\title{
Enhanced photon correlations due to strong laser-atom-cavity coupling
}

\author{
Viorel Ciornea, Profirie Bardetski, and Mihai A. Macove* \\ Institute of Applied Physics, Academy of Sciences of Moldova, Academiei str. 5, MD-2028 Chişinău, Moldova
}

(Dated: August 25, 2021)

\begin{abstract}
We investigate the resonant quantum dynamics of a laser-pumped real or artificial two-level singleatom system embedded in a leaking microcavity. We found that for stronger laser-atom-cavity couplings the generated microcavity photons exhibit larger steady-state correlations. In particular, the second- and third-order photon correlation functions are greater than the corresponding ones obtained for an incoherent light source, respectively. Furthermore, the emitted microcavity photon flux is enhanced in comparison to weaker coupling cases.

PACS numbers: 42.50.Ar, 42.50.Ct, 73.21.La
\end{abstract}

\section{INTRODUCTION}

Correlation functions describing the coherence properties of interacting or noninteracting quantum particles received considerable attention [1-17]. These functions are used almost in all branches of modern physics. Typically, standard sources of incoherent light generate photons possessing a second-order correlation function $g^{(2)}(0)=2[1]$. Very recently, it was shown that photons emitted by a single two-level system ultra-strongcoupled with a thermal optical cavity show photon correlation functions $g^{(2)}(0)>2$ or even smaller than unity exhibiting quantum light features, i.e. sub-Poissonian photon statistics [4]. The effect occurs when the atomcavity coupling rate becomes comparable to the cavity resonance frequency. For such an interaction regime, the counter-rotating terms in the interaction Hamiltonian should be taken into account. Therefore, various novel schemes generating highly correlated light are still important. Particularly, larger photon correlations are useful for a number of practical applications in manybody phenomena with strongly interacting photons [5] as well as in photonic quantum information processing [68]. An ensemble of $N$ collectively interacting few-level atoms via an incoherent electromagnetic field reservoir generates light with $g^{(2)}(0)=4$ or even higher in the steady-state. However, the photon intensity is rather weak [9, 10]. Applying external coherent light sources, one can generate intenser photon fluxes proportional to $N$ or $N^{2}$ with, however, lower second-order photon correlations in the steady-state, i.e. $g^{(2)}(0)<4$ [11, 12]. Furthermore, higher-order photon correlations [13, 14] can be generated in a somehow more complicated setup involving nonlinear crystal superlattices [15]. Finally, correlated photon emission can be achieved from multiatom entangled Rydberg states, for instance [16, 17]. Thus, it becomes intriguing to find alternative ways to generate both an intense steady-state photon flux with enhanced photon-photon correlations.

Here, we demonstrate a scheme capable to generate

*Electronic address: macovei@phys.asm.md a moderately intense and highly correlated photon flux. In particular, the obtained second- and third-order photon correlation functions in the steady-state are several times larger than corresponding ones but for a thermal light source. The scheme is based on pumping a twolevel emitter embedded in a leaking optical microcavity. At moderately strong pumping, i.e. the respective Rabi frequency is larger than the spontaneous and cavity decay rates, respectively, the spontaneous scattered photons into free electromagnetic field modes show the well-known Mollow spectrum [18] modified by the cavity field [19]. Now, if the laser-atom-cavity system is in resonance with the central-band of the Mollow spectrum then the side-bands will contribute to the atommicrocavity quantum dynamics only via the non-secular terms. Under certain conditions, these terms are responsible for enhancing the microcavity mean-photon number as well as the second- and third-order photon correlations in the steady-state. Furthermore, a moderate incoherent pumping of the resonator mode does not modify the photon statistics considerably. Notice the existence of an experiment measuring two- and three-photon correlations in a strongly driven atom-cavity-system for different parameters of interest [20]. This makes our results experimentally attractive.

The article is organized as follows. In Section II we describe the analytical approach and the system of interest, while in Section III, we obtain the corresponding equations of motion and describe the obtained results. The Summary is given in Section IV.

\section{NONLINEAR QUANTUM DYNAMICS OF A PUMPED QUBIT INSIDE A MICROCAVITY}

The Hamiltonian describing a two-level real (or artificial) atomic system possessing the frequency $\omega_{0}$ and interacting with a coherent source of frequency $\omega_{L}$, and embedded in a microcavity of frequency $\omega_{c}$, in a frame rotating at $\omega_{L}$, is:

$$
H=\hbar \Delta a^{\dagger} a+\hbar g\left(a^{\dagger} S^{-}+a S^{+}\right)+\hbar \Omega\left(S^{+}+S^{-}\right),
$$

where we have assumed that $\omega_{0}=\omega_{L}$. In the Hamiltonian (1) the first term describes the cavity free energy 
with $\Delta=\omega_{c}-\omega_{L}$, while the second one characterizes the interaction of the two-level emitter with the microcavity mode via the coupling $g$. The third term considers the qubit's interaction with the laser field with $\Omega$ being the corresponding Rabi frequency. The atomic bare-state operators $S^{+}=|2\rangle\langle 1|$ and $S^{-}=\left[S^{+}\right]^{+}$obey the commutation relations for $\mathrm{su}(2)$ algebra: $\left[S^{+}, S^{-}\right]=2 S_{z}$ and $\left[S_{z}, S^{ \pm}\right]= \pm S^{ \pm}$. Here, $S_{z}=(|2\rangle\langle 2|-| 1\rangle\langle 1|) / 2$ is the bare-state inversion operator. $|2\rangle$ and $|1\rangle$ are the excited and ground state of the qubit, respectively, while $a^{\dagger}$ and $a$ are the creation and the annihilation operator of the electromagnetic field (EMF) in the resonator, and satisfy the standard bosonic commutation relations, i.e., $\left[a, a^{\dagger}\right]=1$, and $[a, a]=\left[a^{\dagger}, a^{\dagger}\right]=0$.

We are interested in the laser dominated regime where $\Omega \gg\{\gamma, \kappa\}$ (here, $\gamma$ and $\kappa$ are the spontaneous and cavity decay rates, respectively) and shall describe our system using the dressed-states formalism [3]: $|1\rangle=$ $(|\overline{1}\rangle+|\overline{2}\rangle) / \sqrt{2}$, and $|2\rangle=(|\overline{2}\rangle-|\overline{1}\rangle) / \sqrt{2}$. Applying this transformation in Eq. (1) with $\Delta=0$, one arrive then at the following dressed-state Hamiltonian in a frame rotating at the Rabi frequency $\Omega$ :

$$
\begin{aligned}
H_{0} & =\hbar g_{0} R_{z}\left(a+a^{\dagger}\right)+\hbar g_{0}\left(R^{+} e^{2 i \Omega t}-R^{-} e^{-2 i \Omega t}\right) \\
& \times\left(a^{\dagger}-a\right) .
\end{aligned}
$$

Here, $g_{0}=g / 2$ while the new quasispin operators, i.e. $R^{+}=|\overline{2}\rangle\langle\overline{1}|, R^{-}=\left[R^{+}\right]^{+}$and $R_{z}=|\overline{2}\rangle\langle\overline{2}|-| \overline{1}\rangle\langle\overline{1}|$ are operating in the dressed-state picture. They obey the following commutation relations: $\left[R^{+}, R^{-}\right]=R_{z}$ and $\left[R_{z}, R^{ \pm}\right]= \pm 2 R^{ \pm}$. One can observe that the Hamiltonian (2) can be separated into a time-independent part and a time-dependent one containing fast oscillating terms. Therefore, the time-dependent part, $H_{f}$, can be regarded as a perturbation to the time-independent part when $\Omega>g_{0}$. One can apply then the transformation: $\bar{H}=-\frac{i}{\hbar} H_{f}(t) \int d t H_{f}(t)[2123]$ to arrive at the final time-independent Hamiltonian characterizing the coherent evolution of the laser-atom-cavity system:

$$
H_{0}=\hbar g_{0} R_{z}\left(a^{\dagger}+a\right)+\hbar \beta R_{z} a^{\dagger} a-\hbar \frac{\beta}{2} R_{z}\left(a^{\dagger 2}+a^{2}\right),
$$

where $\beta=g_{0}^{2} / \Omega$ and $\beta \ll g_{0}$. The nonlinear terms proportional to $\beta$ in Eq. (3) are due to the non-secular contribution and are responsible for squeezed one-atom lasing [21] as well as non-classical EMF coherences [22] in a different related setup.

In the Heisenberg picture, the master equation describing the laser-dressed two-level qubit inside a leaking resonator and damped via the vacuum modes of the surrounding EMF reservoir [3] is:

$$
\begin{aligned}
& \frac{d}{d t}\langle Q(t)\rangle-\frac{i}{\hbar}\left\langle\left[H_{0}, Q\right]\right\rangle=-\Gamma_{0}\left\langle R_{z}\left[R_{z}, Q\right]\right\rangle \\
- & \Gamma\left\{\left\langle R^{+}\left[R^{-}, Q\right]\right\rangle+\left\langle R^{-}\left[R^{+}, Q\right]\right\rangle\right\} \\
- & \kappa(1+\bar{n})\left\langle a^{\dagger}[a, Q]\right\rangle-\kappa \bar{n}\left\langle a\left[a^{\dagger}, Q\right]\right\rangle+H . c . .
\end{aligned}
$$

Here, in general, for the non-Hermitian atomic or EMF operators $Q$, the H.c. terms should be evaluated without conjugating $Q$, i.e. by replacing $Q^{+}$with $Q$ in

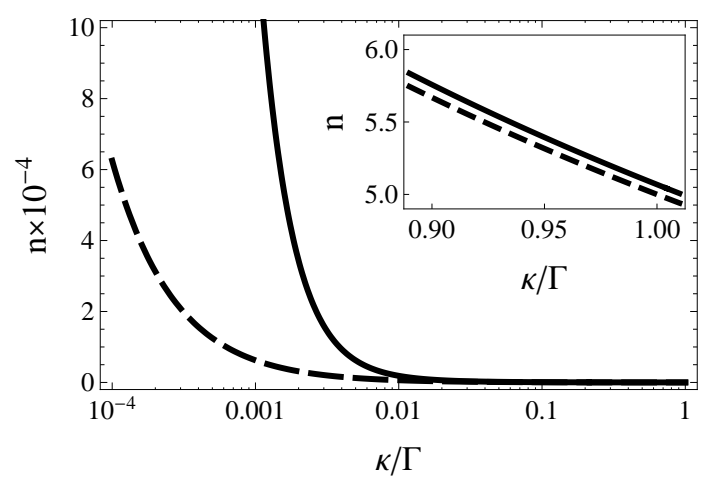

FIG. 1: The steady-state dependences of the microcavity mean-photon number $n=\left\langle a^{\dagger} a\right\rangle_{s}$ versus the parameter $\kappa / \Gamma$. Dashed and solid curves are for $\beta / \Gamma=0$ and 0.1 , respectively. The inset shows the same behaviour for a particular range of $\kappa / \Gamma$. Here, $\bar{n}=0$ and $g_{0} / \Gamma=5$.

the Hermitian conjugate parts. Further, $\Gamma_{0}=\gamma / 4$ and $\Gamma=\left(\gamma+\gamma_{d}\right) / 4$ with $2 \gamma$ being the single-atom spontaneous decay rate, while $\gamma_{d}$ is the qubit dephasing rate. Finally, $\bar{n}$ is the microcavity mean-photon number due to incoherent pumping of the cavity mode. This is achieved via pumping of the microcavity mode with a broadband laser field with its spectral width larger than the resonator decay rate $\kappa$, respectively. Note that in Eq. (4), we have performed the secular approximation in the spontaneous emission damping [12].

In the following section, we shall describe the microcavity photon statistics via the second- and third-order photon correlation functions.

\section{SECOND- AND THIRD-ORDER PHOTON CORRELATION FUNCTIONS}

The equations of motion for the variables of interest can be easily obtained from the Master Equation (4). For instance, the steady-state value of the mean-photon number, i.e. $\left\langle a^{\dagger} a\right\rangle_{s}$, can be extracted from the following system of linear steady-state equations:

$$
\begin{aligned}
0 & =2 \kappa\left\langle a^{\dagger} a\right\rangle_{s}+i g_{0}\left\langle R_{z} a^{\dagger}\right\rangle_{s}-i g_{0}\left\langle R_{z} a\right\rangle_{s} \\
& -i \beta\left\langle R_{z} a^{\dagger 2}\right\rangle_{s}+i \beta\left\langle R_{z} a^{2}\right\rangle_{s}-2 \kappa \bar{n}, \\
0 & =(\kappa+4 \Gamma)\left\langle R_{z} a^{\dagger}\right\rangle_{s}-i \beta\left\langle a^{\dagger}\right\rangle_{s}+i \beta\langle a\rangle_{s}-i g_{0}, \\
0 & =(2 \kappa+4 \Gamma)\left\langle R_{z} a^{\dagger 2}\right\rangle_{s}-2 i g_{0}\left\langle a^{\dagger}\right\rangle_{s}-2 i \beta\left\langle a^{\dagger 2}\right\rangle_{s} \\
& +i \beta\left(1+2\left\langle a^{\dagger} a\right\rangle_{s}\right), \\
0 & =\kappa\left\langle a^{\dagger}\right\rangle_{s}-i \beta\left\langle R_{z} a^{\dagger}\right\rangle_{s}+i \beta\left\langle R_{z} a\right\rangle_{s}, \\
0 & =2 \kappa\left\langle a^{\dagger 2}\right\rangle_{s}-2 i g_{0}\left\langle R_{z} a^{\dagger}\right\rangle_{s}-2 i \beta\left\langle R_{z} a^{\dagger 2}\right\rangle_{s} \\
& +2 i \beta\left\langle R_{z} a^{\dagger} a\right\rangle_{s}, \\
0 & =(2 \kappa+4 \Gamma)\left\langle R_{z} a^{\dagger} a\right\rangle_{s}+i g_{0}\left\langle a^{\dagger}\right\rangle_{s}-i g_{0}\langle a\rangle_{s} \\
& -i \beta\left\langle a^{\dagger 2}\right\rangle_{s}+i \beta\left\langle a^{2}\right\rangle_{s} .
\end{aligned}
$$

In the system of equations (5), and throughout the paper, we used the fact that the dressed-state inversion is zero 


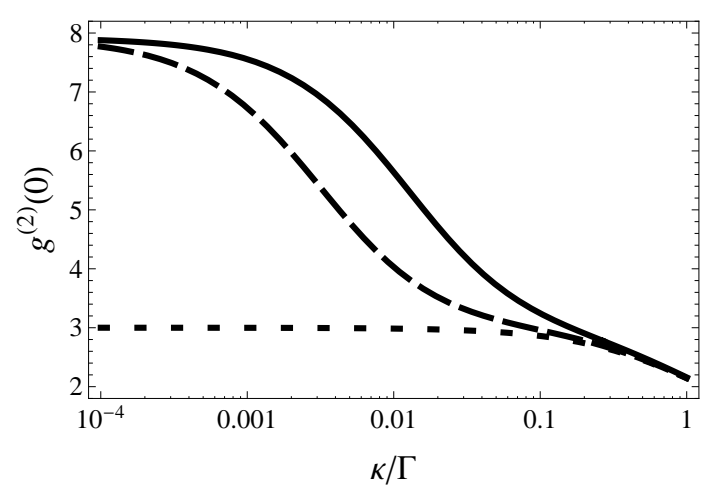

FIG. 2: The steady-state dependences of the microcavity second-order photon correlation function $g^{(2)}(0)$ versus the parameter $\kappa / \Gamma$. Short-dashed, long-dashed and solid curves are for $\beta / \Gamma=0,0.05$, and 0.1 , respectively. Other parameters are: $\bar{n}=0$ and $g_{0} / \Gamma=5$.

in the steady-state, i.e. $\left\langle R_{z}\right\rangle_{s}=0$, as well as the trivial condition $R_{z}^{2}=1$ which is the case for a single-atom system. By completing the system of equations Eq. (5) with the respective H.c. equations, it takes a closed-form and can be exactly solved. In particular, one of the solution of (5) represents the steady-state mean-photon number in the microcavity mode, namely:

$$
\left\langle a^{\dagger} a\right\rangle_{s}=\bar{n}+\frac{g_{0}^{2}}{\kappa(\kappa+4 \Gamma)}+\frac{8 g_{0}^{2}+\kappa(\kappa+4 \Gamma)(1+2 \bar{n})}{2 \kappa^{2}(\kappa+2 \Gamma)(\kappa+4 \Gamma)} \beta^{2} .
$$

One can observe here, that the mean-photon number in the steady-state is enhanced due to both the incoherent pumping and the nonlinear contribution proportional to $\beta^{2}$. This is clearly seen in Figure (1), where we plotted the microcavity mean-photon number, $n=\left\langle a^{\dagger} a\right\rangle_{s}$, as a function of different relevant parameters. For $\kappa / \Gamma>1$, the photon number in the steady-state goes to $\bar{n}$ as the bad-cavity limit is achieved.

Further, we focus on the degree of second- and thirdorder coherences of microcavity photons defined, respectively, as [1, 2]:

$$
g^{(2)}(0)=\frac{\left\langle a^{\dagger 2} a^{2}\right\rangle_{s}}{\left\langle a^{\dagger} a\right\rangle_{s}^{2}} \text {, and } g^{(3)}(0)=\frac{\left\langle a^{\dagger 3} a^{3}\right\rangle_{s}}{\left\langle a^{\dagger} a\right\rangle_{s}^{3}}
$$

The unnormalized k-order correlation function $\left\langle a^{\dagger k} a^{k}\right\rangle_{s}$ describes the probability of a k-photon detection, simultaneously. To obtain the steady-state expressions for correlation functions given in Eq. (7), the system of equations (5D) have to be completed with additional equations obtained with the help of Eq. (44). In particular, the unnormalized steady-state second-order photon correlation function can be obtained from the following system of equations (and the corresponding H.c. expressions):

$$
\begin{aligned}
0 & =4 \kappa\left\langle a^{\dagger 2} a^{2}\right\rangle_{s}+2 i g_{0}\left\langle R_{z} a^{\dagger 2} a\right\rangle_{s}-2 i g_{0}\left\langle R_{z} a^{\dagger} a^{2}\right\rangle_{s} \\
& -i \beta\left(\left\langle R_{z} a^{\dagger 2}\right\rangle_{s}+2\left\langle R_{z} a^{\dagger 3} a\right\rangle_{s}\right. \\
& \left.-\left\langle R_{z} a^{2}\right\rangle_{s}-2\left\langle R_{z} a^{\dagger} a^{3}\right\rangle_{s}\right)-8 \kappa \bar{n}\left\langle a^{\dagger} a\right\rangle_{s}, \\
0 & =(3 \kappa+4 \Gamma)\left\langle R_{z} a^{\dagger 2} a\right\rangle_{s}+i g_{0}\left\langle a^{\dagger 2}\right\rangle_{s}-2 i g_{0}\left\langle a^{\dagger} a\right\rangle_{s} \\
& -i \beta\left(\left\langle a^{\dagger 2} a\right\rangle_{s}+\left\langle a^{\dagger 3}\right\rangle_{s}-\langle a\rangle_{s}-2\left\langle a^{\dagger} a^{2}\right\rangle_{s}\right) \\
& -4 \kappa \bar{n}\left\langle R_{z} a^{\dagger}\right\rangle_{s}, \\
0 & =(4 \kappa+4 \Gamma)\left\langle R_{z} a^{\dagger 3} a\right\rangle_{s}+i g_{0}\left\langle a^{\dagger 3}\right\rangle_{s}-3 i g_{0}\left\langle a^{\dagger 2} a\right\rangle_{s} \\
& -i \beta\left(2\left\langle a^{\dagger 3} a\right\rangle_{s}+\left\langle a^{\dagger 4}\right\rangle_{s}-3\left\langle a^{\dagger 2} a^{2}\right\rangle_{s}-3\left\langle a^{\dagger} a\right\rangle_{s}\right) \\
& -6 \kappa \bar{n}\left\langle R_{z} a^{\dagger 2}\right\rangle_{s}, \\
0 & =3 \kappa\left\langle a^{\dagger} a^{2}\right\rangle_{s}+2 i g_{0}\left\langle R_{z} a^{\dagger} a\right\rangle_{s}-i g_{0}\left\langle R_{z} a^{2}\right\rangle_{s} \\
& +i \beta\left(\left\langle R_{z} a^{\dagger} a^{2}\right\rangle_{s}-\left\langle R_{z} a^{\dagger}\right\rangle_{s}\right. \\
& \left.-2\left\langle R_{z} a^{\dagger 2} a\right\rangle_{s}+\left\langle R_{z} a^{3}\right\rangle_{s}\right)-4 \kappa \bar{n}\langle a\rangle_{s}, \\
0 & =3 \kappa\left\langle a^{\dagger 3}\right\rangle_{s}-3 i g_{0}\left\langle R_{z} a^{\dagger 2}\right\rangle_{s} \\
& +3 i \beta\left(\left\langle R_{z} a^{\dagger 2} a\right\rangle_{s}+\left\langle R_{z} a^{\dagger}\right\rangle_{s}-\left\langle R_{z} a^{\dagger 3}\right\rangle_{s}\right), \\
0 & =4 \kappa\left\langle a^{\dagger 4}\right\rangle_{s}-4 i g_{0}\left\langle R_{z} a^{\dagger 3}\right\rangle_{s} \\
& +2 i \beta\left(2\left\langle R_{z} a^{\dagger 3} a\right\rangle_{s}+3\left\langle R_{z} a^{\dagger 2}\right\rangle_{s}-2\left\langle R_{z} a^{\dagger 4}\right\rangle_{s}\right), \\
0 & =4 \kappa\left\langle a^{\dagger 3} a\right\rangle_{s}+i g_{0}\left\langle R_{z} a^{\dagger 3}\right\rangle_{s}-3 i g_{0}\left\langle R_{z} a^{\dagger 2} a\right\rangle_{s} \\
& -i \beta\left(2\left\langle R_{z} a^{\dagger 3} a\right\rangle_{s}+\left\langle R_{z} a^{\dagger 4}\right\rangle_{s}\right. \\
& \left.-3\left\langle R_{z} a^{\dagger 2} a^{2}\right\rangle_{s}-3\left\langle R_{z} a^{\dagger} a\right\rangle_{s}\right)-6 \kappa \bar{n}\left\langle a^{\dagger 2}\right\rangle_{s}, \\
0 & =(3 \kappa+4 \Gamma)\left\langle R_{z} a^{\dagger 3}\right\rangle_{s}-3 i g_{0}\left\langle a^{\dagger 2}\right\rangle_{s} \\
& +3 i \beta\left(\left\langle a^{\dagger 2} a\right\rangle_{s}+\left\langle a^{\dagger}\right\rangle_{s}-\left\langle a^{\dagger 3}\right\rangle_{s}\right), \\
0 & =(4 \kappa+4 \Gamma)\left\langle R_{z} a^{\dagger 4}\right\rangle_{s}-4 i g_{0}\left\langle a^{\dagger 3}\right\rangle_{s} \\
& +2 i \beta\left(2\left\langle a^{\dagger 3} a\right\rangle_{s}+3\left\langle a^{\dagger 2}\right\rangle_{s}-2\left\langle a^{\dagger 4}\right\rangle_{s}\right), \\
0 & =(4 \kappa+4 \Gamma)\left\langle R_{z} a^{\dagger 2} a^{2}\right\rangle_{s}+2 i g_{0}\left\langle a^{\dagger 2} a\right\rangle_{s}-2 i g_{0}\left\langle a^{\dagger} a^{2}\right\rangle_{s} \\
& -i \beta\left(\left\langle a^{\dagger 2}\right\rangle_{s}+2\left\langle a^{\dagger 3} a\right\rangle_{s}\right. \\
& \left.-\left\langle a^{2}\right\rangle_{s}-2\left\langle a^{\dagger} a^{3}\right\rangle_{s}\right)-8 \kappa \bar{n}\left\langle R_{z} a^{\dagger} a\right\rangle_{s} . \\
& =18
\end{aligned}
$$

The equation of motions required to obtain the thirdorder correlation function are given in the Appendix B.

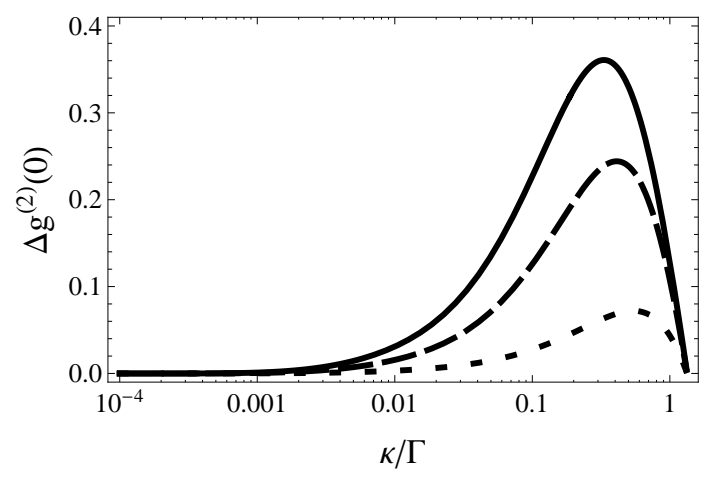

FIG. 3: The variance of the second-order correlation functions $\Delta g^{(2)}(0)=\left.g^{(2)}(0)\right|_{\bar{n} \neq 0}-\left.g^{(2)}(0)\right|_{\bar{n}=0}$ as a function of $\kappa / \Gamma$. Short-dashed, long-dashed and solid lines are for $\bar{n}=1,5$, and 10 , respectively. Here, $g_{0} / \Gamma=5$ and $\beta / \Gamma=0.05$. 


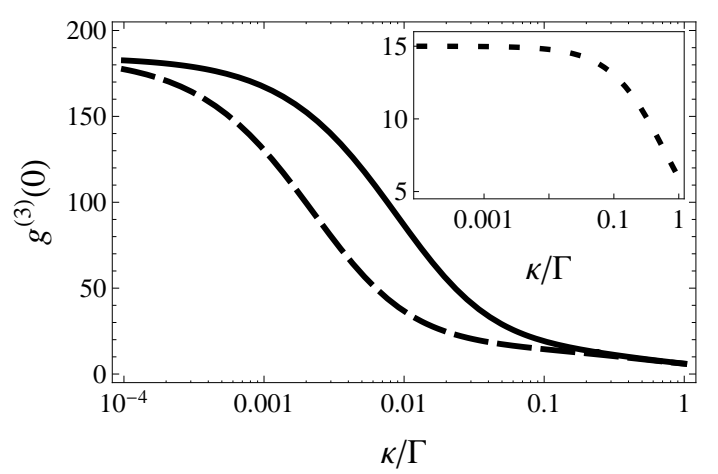

FIG. 4: The steady-state dependences of the microcavity third-order photon coherence function $g^{(3)}(0)$ as a function of $\kappa / \Gamma$. Solid and long-dashed curves are for $\beta / \Gamma=0.1$ and 0.05 , respectively. The inset shows the case of $\beta / \Gamma=0$. Other parameters are: $\bar{n}=0$ and $g_{0} / \Gamma=5$.

Taking into account the systems of equations (5) and (8), one can obtain the steady-state expression for the second-order coherence function, that is:

$$
g^{(2)}(0)=\frac{(\kappa+2 \Gamma)(\kappa+4 \Gamma)}{3(\kappa+\Gamma)^{2}(3 \kappa+4 \Gamma)^{2}} \frac{A+B \beta^{2}+C \beta^{4}}{\tilde{A}+\tilde{B} \beta^{2}+\tilde{C} \beta^{4}},
$$

where

$$
\begin{aligned}
& A=A_{0}+A_{1} \bar{n}+A_{2} \bar{n}^{2}, \quad \tilde{A}=\tilde{A}_{0}+\tilde{A}_{1} \bar{n}+\tilde{A}_{2} \bar{n}^{2}, \\
& B=B_{0}+B_{1} \bar{n}+B_{2} \bar{n}^{2}, \quad \tilde{B}=\tilde{B}_{0}+\tilde{B}_{1} \bar{n}+\tilde{B}_{2} \bar{n}^{2}, \\
& C=C_{0}+C_{1} \bar{n}+C_{2} \bar{n}^{2}, \tilde{C}=\tilde{C}_{0}+\tilde{C}_{1} \bar{n}+\tilde{C}_{2} \bar{n}^{2},
\end{aligned}
$$

while the missing parameters are given in Eqs. (A1), in the Appendix A. Here, again, one can observe that the nonlinear term $\beta$ modifies the second-order photon correlation. In particular, when $\beta=0$ one obtains:

$$
g^{(2)}(0)=2+\frac{g_{0}^{4}(4 \Gamma-3 \kappa)}{(4 \Gamma+3 \kappa)\left(g_{0}^{2}+\kappa(\kappa+4 \Gamma) \bar{n}\right)^{2}},
$$

while for $\left\{g_{0}, \beta\right\}=0$ one has $g^{(2)}(0)=2$, that is, we recover the incoherent-source result for a second-order correlation function. For smaller values of $\kappa / \Gamma$ and $\beta \neq 0$, the second-order coherence function tends to a constant value:

$$
\lim _{\kappa \rightarrow 0} g^{(2)}(0)=\frac{95}{12} \approx 7.91,
$$

while for $\beta=0$ and $g_{0} \neq 0$ we have:

$$
\lim _{\kappa \rightarrow 0} g^{(2)}(0)=3 \text {. }
$$

These behaviors are shown in Fig. (2), plotted with the help of Eq. (9). Thus, for particular values of involved parameters one can obtain larger photon correlations, i.e. $g^{(2)}(0) \gg 2$, as well as bigger photon numbers (see Fig. 2 and Fig. 1, respectively) due to nonlinear terms proportional to $\beta$ in the interaction Hamiltonian (3). To elucidate the role played by the incoherent pumping, in Fig. (3), we depict the difference

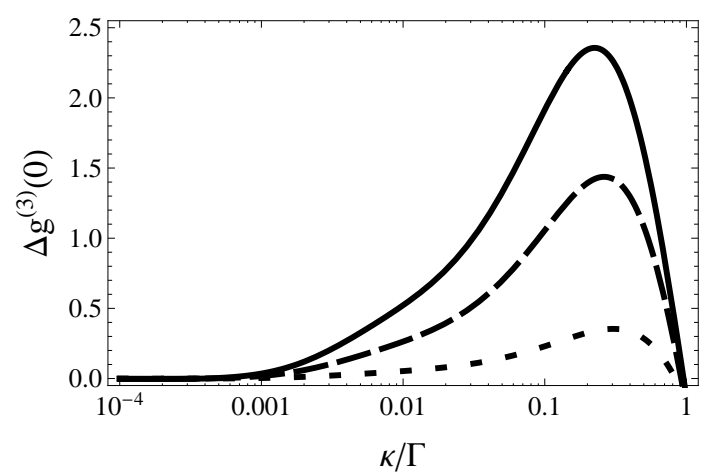

FIG. 5: The same as in Fig. (3), but for $\Delta g^{(3)}(0)=$ $\left.g^{(3)}(0)\right|_{\bar{n} \neq 0}-\left.g^{(3)}(0)\right|_{\bar{n}=0}$.

$\Delta g^{(2)}(0)=\left.g^{(2)}(0)\right|_{\bar{n} \neq 0}-\left.g^{(2)}(0)\right|_{\bar{n}=0}$ as a function of $\kappa / \Gamma$. For very small values of $\kappa / \Gamma$, a moderate incoherent pumping almost does not affect the photon coherences, while when the ratio $\kappa / \Gamma$ increases the photon statistics modifies accordingly (see Fig. 3 and Eqs. 9 and 10).

In order to understand that the photon correlations indeed are enhanced due to the nonlinearity characterized by $\beta$, in Fig. (4), we plot the third-order microcavity photon correlation function $g^{(3)}(0)$ (with the help of the corresponding equations, i.e. Eqs. (B1), given in the Appendix B). Remarkable, the third-order photon correlations are enhanced as well due to presence of $\beta$. In particular, for an incoherent bath, i.e. $g_{0}=0, g^{(3)}(0)=6$ while for $\beta / \Gamma=0$ the third-order steady-state photon coherence function is given by the expression:

$$
\begin{aligned}
g^{(3)}(0) & =6+\frac{12 g_{0}^{6} \kappa(5 \kappa-12 \Gamma)(5 \kappa+4 \Gamma)^{-1}}{(3 \kappa+4 \Gamma)\left(g_{0}^{2}+\kappa(\kappa+4 \Gamma) \bar{n}\right)^{3}} \\
& +\frac{9 g_{0}^{4}(-3 \kappa+4 \Gamma)}{(3 \kappa+4 \Gamma)\left(g_{0}^{2}+\kappa(\kappa+4 \Gamma) \bar{n}\right)^{2}} .
\end{aligned}
$$

In general, i.e. for $\beta \neq 0$, the expression for $g^{(3)}(0)$ is too complicated and it is not shown here. However, for smaller values of $\kappa / \Gamma$ and $\beta \neq 0$ one has:

$$
\lim _{\kappa \rightarrow 0} g^{(3)}(0)=\frac{33203}{180} \approx 184.46 .
$$

If $\beta=0$ and $g_{0} \neq 0$ we have:

$$
\lim _{\kappa \rightarrow 0} g^{(3)}(0)=15 \text {. }
$$

Thus, in both limits described by expressions (14]15), the third-order correlation function is bigger than the corresponding one but for an incoherent-type light source, i.e. $g^{(3)}(0)>6$ (see, also, Fig. (4). The influence of a moderate incoherent pumping into the cavity mode on third-order photon statistics is shown in Fig. (5). Similar to Fig. (3), very small values of $\kappa / \Gamma$ do not modify the third-order photon correlations in presence of incoherent photons $\bar{n} . g^{(3)}(0)$ slightly changes with increasing $\bar{n}$ and $\kappa / \Gamma$. Stronger incoherent pumping will lead to a photon 
statistics which is typical for an incoherent-light source. Furthermore, the photon statistics does not depend on $g_{0}$ for $\{\bar{n}, \beta\}=0$ (see, for instance, Eq. 10] and Eq. 13). Finally, one can conjecture that even higher-order photon correlation functions will behave similarly to those shown in Fig. (2) - Fig. (5) with, however, larger magnitudes.

\section{SUMMARY}

In summary, we have investigated the correlations functions of microcavity photons generated due to coherent interaction of a two-level qubit with an external laser field as well as a microcavity mode and in the presence of both the spontaneous emission and cavity damping, respectively. In addition, the cavity mode is pumped incoherently. In the intense-field limit, i.e. the involved Rabi frequency is larger than the spontaneous and cavity decay rates, respectively, we found enhanced second- and third-order photon correlations. These correlation functions are larger than the corresponding ones but for an incoherent-light source. The photon correlation enhancement is due to the non-secular contribution in the coherent dressed-state Hamiltonian describing the pumped atom interacting with the microcavity mode. This contribution is relevant for stronger laser-atom-cavity interactions.

\section{Acknowledgments}

We are grateful to financial support via the research grant Nr. 13.820.05.07/GF.

\section{Appendix A: The parameters entering in the second-order photon correlation function}

Here are the parameters entering in Eq. (9).

$$
\begin{aligned}
A_{0} & =36 g_{0}^{4} \kappa^{2}(\Gamma+\kappa)^{2}(2 \Gamma+\kappa)(4 \Gamma+3 \kappa), \\
A_{1} & =48 g_{0}^{2} \kappa^{3}(\Gamma+\kappa)^{2}(2 \Gamma+\kappa)(4 \Gamma+3 \kappa)^{2}, \\
A_{2} & =24 \kappa^{4}(\Gamma+\kappa)^{2}(2 \Gamma+\kappa)(4 \Gamma+\kappa)(4 \Gamma+3 \kappa)^{2}, \\
\tilde{A}_{0} & =4 g_{0}^{4} \kappa^{2}(2 \Gamma+\kappa)^{2}, \\
\tilde{A}_{1} & =8 g_{0}^{2} \kappa^{3}(2 \Gamma+\kappa)^{2}(4 \Gamma+\kappa), \\
\tilde{A}_{2} & =4 \kappa^{4}(2 \Gamma+\kappa)^{2}(4 \Gamma+\kappa)^{2}, \\
B_{0} & =\kappa(\Gamma+\kappa)\left(3 \kappa^{2}(\Gamma+\kappa)(4 \Gamma+\kappa)(4 \Gamma+3 \kappa)^{2}\right. \\
& +32 g_{0}^{4}\left(50 \Gamma^{2}+74 \Gamma \kappa+27 \kappa^{2}\right) \\
& \left.+4 g_{0}^{2} \kappa(4 \Gamma+3 \kappa)\left(100 \Gamma^{2}+139 \Gamma \kappa+45 \kappa^{2}\right)\right), \\
B_{1} & =4 \kappa^{2}(\Gamma+\kappa)(4 \Gamma+3 \kappa)\left(g _ { 0 } ^ { 2 } \left(392 \Gamma^{2}+614 \Gamma \kappa\right.\right. \\
& \left.\left.+234 \kappa^{2}\right)+9 \kappa(\Gamma+\kappa)(4 \Gamma+\kappa)(4 \Gamma+3 \kappa)\right),
\end{aligned}
$$

$$
\begin{aligned}
B_{2} & =60 \kappa^{3}(\Gamma+\kappa)^{2}(4 \Gamma+\kappa)(4 \Gamma+3 \kappa)^{2} \\
\tilde{B}_{0} & =4 \kappa g_{0}^{2}(2 \Gamma+\kappa)\left(8 g_{0}^{2}+\kappa(4 \Gamma+\kappa)\right) \\
\tilde{B}_{1} & =4 \kappa^{2}(2 \Gamma+\kappa)(4 \Gamma+\kappa)\left(10 g_{0}^{2}+\kappa(4 \Gamma+\kappa)\right) \\
\tilde{B}_{2} & =8 \kappa^{3}(2 \Gamma+\kappa)(4 \Gamma+\kappa)^{2} \\
C_{0} & =9 \kappa^{2}(\Gamma+\kappa)(4 \Gamma+\kappa)(4 \Gamma+3 \kappa)^{2} \\
& +16 g_{0}^{4}\left(190 \Gamma^{2}+289 \Gamma \kappa+108 \kappa^{2}\right) \\
& +4 g_{0}^{2} \kappa(4 \Gamma+3 \kappa)\left(220 \Gamma^{2}+319 \Gamma \kappa+108 \kappa^{2}\right) \\
C_{1} & =4 \kappa(4 \Gamma+3 \kappa)(9 \kappa(\Gamma+\kappa)(4 \Gamma+\kappa)(4 \Gamma+3 \kappa) \\
& \left.+g_{0}^{2}\left(440 \Gamma{ }^{2}+638 \Gamma \kappa+216 \kappa^{2}\right)\right) \\
C_{2} & =36 \kappa^{2}(\Gamma+\kappa)(4 \Gamma+\kappa)(4 \Gamma+3 \kappa)^{2}, \\
\tilde{C}_{0} & =\left(8 g_{0}^{2}+\kappa(4 \Gamma+\kappa)\right)^{2} \\
\tilde{C}_{1} & =4 \kappa(4 \Gamma+\kappa)\left(8 g_{0}^{2}+\kappa(4 \Gamma+\kappa)\right), \\
\tilde{C}_{2} & =4 \kappa^{2}(4 \Gamma+\kappa)^{2} .
\end{aligned}
$$

\section{Appendix B: Equations of motion for the third-order photon coherences}

To obtain the third-order correlation function $g^{(3)}(0)$ the system of linear equations (5) \& (8) should be completed with the following equations of motion (and the corresponding H.c. equations):

$$
\begin{aligned}
0= & 6 \kappa\left\langle a^{\dagger 3} a^{3}\right\rangle_{s}+3 i g_{0}\left\langle R_{z} a^{\dagger 3} a^{2}\right\rangle_{s}-3 i g_{0}\left\langle R_{z} a^{\dagger 2} a^{3}\right\rangle_{s} \\
& -3 i \beta\left(\left\langle R_{z} a^{\dagger 4} a^{2}\right\rangle_{s}+\left\langle R_{z} a^{\dagger 3} a\right\rangle_{s}\right. \\
& \left.-\left\langle R_{z} a^{\dagger 2} a^{4}\right\rangle_{s}-\left\langle R_{z} a^{\dagger} a^{3}\right\rangle_{s}\right)-18 \kappa \bar{n}\left\langle a^{\dagger 2} a^{2}\right\rangle_{s}, \\
0= & 6 \kappa+4 \Gamma)\left\langle R_{z} a^{\dagger 3} a^{3}\right\rangle_{s}+3 i g_{0}\left\langle a^{\dagger 3} a^{2}\right\rangle_{s} \\
& -3 i g_{0}\left\langle a^{\dagger 2} a^{3}\right\rangle_{s}-3 i \beta\left(\left\langle a^{\dagger 4} a^{2}\right\rangle_{s}+\left\langle a^{\dagger 3} a\right\rangle_{s}\right. \\
& \left.-\left\langle a^{\dagger 2} a^{4}\right\rangle_{s}-\left\langle a^{\dagger} a^{3}\right\rangle_{s}\right)-18 \kappa \bar{n}\left\langle R_{z} a^{\dagger 2} a^{2}\right\rangle_{s}, \\
0= & (5 \kappa+4 \Gamma)\left\langle R_{z} a^{\dagger 3} a^{2}\right\rangle_{s}+2 i g_{0}\left\langle a^{\dagger 3} a\right\rangle_{s}-3 i g_{0}\left\langle a^{\dagger 2} a^{2}\right\rangle_{s} \\
& -i \beta\left(\left\langle a^{\dagger 3} a^{2}\right\rangle_{s}+\left\langle a^{\dagger 3}\right\rangle_{s}+2\left\langle a^{\dagger 4} a\right\rangle_{s}\right. \\
& \left.-3\left\langle a^{\dagger 2} a^{3}\right\rangle_{s}-3\left\langle a^{\dagger} a^{2}\right\rangle_{s}\right)-12 \kappa \bar{n}\left\langle R_{z} a^{\dagger 2} a\right\rangle_{s}, \\
0= & 5 \kappa\left\langle a^{\dagger 3} a^{2}\right\rangle_{s}+2 i g_{0}\left\langle R_{z} a^{\dagger 3} a\right\rangle_{s}-3 i g_{0}\left\langle R_{z} a^{\dagger 2} a^{2}\right\rangle_{s} \\
& -i \beta\left(\left\langle R_{z} a^{\dagger 3} a^{2}\right\rangle_{s}+\left\langle R_{z} a^{\dagger 3}\right\rangle_{s}+2\left\langle R_{z} a^{\dagger 4} a\right\rangle_{s}\right. \\
& \left.-3\left\langle R_{z} a^{\dagger 2} a^{3}\right\rangle_{s}-3\left\langle R_{z} a^{\dagger} a^{2}\right\rangle_{s}\right)-12 \kappa \bar{n}\left\langle a^{\dagger 2} a\right\rangle_{s}, \\
0= & (6 \kappa+4 \Gamma)\left\langle R_{z} a^{\dagger 4} a^{2}\right\rangle_{s}+2 i g_{0}\left\langle a^{\dagger 4} a\right\rangle_{s}-4 i g_{0}\left\langle a^{\dagger 3} a^{2}\right\rangle_{s} \\
& -i \beta\left(2\left\langle a^{\dagger 4} a^{2}\right\rangle_{s}+\left\langle a^{\dagger 4}\right\rangle_{s}+2\left\langle a^{\dagger 5} a\right\rangle_{s}\right. \\
& \left.-4\left\langle a^{\dagger 3} a^{3}\right\rangle_{s}-6\left\langle a^{\dagger 2} a^{2}\right\rangle_{s}\right)-16 \kappa \bar{n}\left\langle R_{z} a^{\dagger 3} a\right\rangle_{s}, \\
0= & 6 \kappa\left\langle a^{\dagger 4} a^{2}\right\rangle_{s}+2 i g_{0}\left\langle R_{z} a^{\dagger 4} a\right\rangle_{s}-4 i g_{0}\left\langle R_{z} a^{\dagger 3} a^{2}\right\rangle_{s} \\
& -i \beta\left(2\left\langle R_{z} a^{\dagger 4} a^{2}\right\rangle_{s}+\left\langle R_{z} a^{\dagger 4}\right\rangle_{s}+2\left\langle R_{z} a^{\dagger 5} a\right\rangle_{s}\right. \\
& \left.-4\left\langle R_{z} a^{\dagger 3} a^{3}\right\rangle_{s}-6\left\langle R_{z} a^{\dagger 2} a^{2}\right\rangle_{s}\right)-16 \kappa \bar{n}\left\langle a^{\dagger 3} a\right\rangle_{s}, \\
0= & (5 \kappa+4 \Gamma)\left\langle R_{z} a^{\dagger 4} a\right\rangle_{s}+i g_{0}\left\langle a^{\dagger 4}\right\rangle_{s}-4 i g_{0}\left\langle a^{\dagger 3} a\right\rangle_{s} \\
& -i \beta\left(3\left\langle a^{\dagger 4} a\right\rangle_{s}+\left\langle a^{\dagger 5}\right\rangle_{s}-4\left\langle a^{\dagger 3} a^{2}\right\rangle_{s}-6\left\langle a^{\dagger 2} a\right\rangle_{s}\right), \\
& -8 \kappa \bar{n}\left\langle R_{z} a^{\dagger 3}\right\rangle_{s},
\end{aligned}
$$




$$
\begin{aligned}
0= & 5 \kappa\left\langle a^{\dagger 4} a\right\rangle_{s}+i g_{0}\left\langle R_{z} a^{\dagger 4}\right\rangle_{s}-4 i g_{0}\left\langle R_{z} a^{\dagger 3} a\right\rangle_{s} \\
& -i \beta\left(3\left\langle R_{z} a^{\dagger 4} a\right\rangle_{s}+\left\langle R_{z} a^{\dagger 5}\right\rangle_{s}\right. \\
& \left.-4\left\langle R_{z} a^{\dagger 3} a^{2}\right\rangle_{s}-6\left\langle R_{z} a^{\dagger 2} a\right\rangle_{s}\right)-8 \kappa \bar{n}\left\langle a^{\dagger 3}\right\rangle_{s}, \\
0= & (6 \kappa+4 \Gamma)\left\langle R_{z} a^{\dagger 5} a\right\rangle_{s}+i g_{0}\left\langle a^{\dagger 5}\right\rangle_{s}-5 i g_{0}\left\langle a^{\dagger 4} a\right\rangle_{s} \\
& -i \beta\left(4\left\langle a^{\dagger 5} a\right\rangle_{s}+\left\langle a^{\dagger 6}\right\rangle_{s}-5\left\langle a^{\dagger 4} a^{2}\right\rangle_{s}-10\left\langle a^{\dagger 3} a\right\rangle_{s}\right) \\
& -10 \kappa \bar{n}\left\langle R_{z} a^{\dagger 4}\right\rangle_{s}, \\
0= & 6 \kappa\left\langle a^{\dagger 5} a\right\rangle_{s}+i g_{0}\left\langle R_{z} a^{\dagger 5}\right\rangle_{s}-5 i g_{0}\left\langle R_{z} a^{\dagger 4} a\right\rangle_{s} \\
& -i \beta\left(4\left\langle R_{z} a^{\dagger 5} a\right\rangle_{s}+\left\langle R_{z} a^{\dagger 6}\right\rangle_{s}\right. \\
& \left.-5\left\langle R_{z} a^{\dagger 4} a^{2}\right\rangle_{s}-10\left\langle R_{z} a^{\dagger 3} a\right\rangle_{s}\right)-10 \kappa \bar{n}\left\langle a^{\dagger 4}\right\rangle_{s}, \\
0= & (5 \kappa+4 \Gamma)\left\langle R_{z} a^{\dagger 5}\right\rangle_{s}-5 i g_{0}\left\langle a^{\dagger 4}\right\rangle_{s} \\
& -i \beta\left(5\left\langle a^{\dagger 5}\right\rangle_{s}-5\left\langle a^{\dagger 4} a\right\rangle_{s}-10\left\langle a^{\dagger 3}\right\rangle_{s}\right), \\
0= & 5 \kappa\left\langle a^{\dagger 5}\right\rangle_{s}-5 i g_{0}\left\langle R_{z} a^{\dagger 4}\right\rangle_{s} \\
& -i \beta\left(5\left\langle R_{z} a^{\dagger 5}\right\rangle_{s}-5\left\langle R_{z} a^{\dagger 4} a\right\rangle_{s}-10\left\langle R_{z} a^{\dagger 3}\right\rangle_{s}\right), \\
0= & (6 \kappa+4 \Gamma)\left\langle R_{z} a^{\dagger 6}\right\rangle_{s}-6 i g_{0}\left\langle a^{\dagger 5}\right\rangle_{s} \\
& -i \beta\left(6\left\langle a^{\dagger 6}\right\rangle_{s}-6\left\langle a^{\dagger 5} a\right\rangle_{s}-15\left\langle a^{\dagger 4}\right\rangle_{s}\right), \\
0= & 6 \kappa\left\langle a^{\dagger 6}\right\rangle_{s}-6 i g_{0}\left\langle R_{z} a^{\dagger 5}\right\rangle_{s} \\
& -i \beta\left(6\left\langle R_{z} a^{\dagger 6}\right\rangle_{s}-6\left\langle R_{z} a^{\dagger 5} a\right\rangle_{s}-15\left\langle R_{z} a^{\dagger 4}\right\rangle_{s}\right) .
\end{aligned}
$$

[1] R. J. Glauber, Phys. Rev. 130, 2529 (1963).

[2] R. J. Glauber, Rev. Mod. Phys. 78, 1267 (2006).

[3] M. O. Scully and M. S. Zubairy, Quantum Optics (Cambridge University Press, Cambridge, 1997).

[4] A. Ridolfo, S. Savasta, M. Hartmann, Phys. Rev. Lett. 110, 163601 (2013).

[5] D. E. Chang, V. Gritsev, G. Morigi, V. Vuletic, M. D. Lukin, E. A. Demler, Nature Phys. 4, 884 (2008).

[6] I. Friedler, D. Petrosyan, M. Fleischhauer, G. Kurizki, Phys. Rev. A 72, 043803 (2005).

[7] A. V. Gorshkov, J. Otterbach, M. Fleischhauer, Th. Pohl, M. D. Lukin, Phys. Rev. Lett. 107, 133602 (2011).

[8] A. I. Lvovsky, B. C. Sanders, W. Tittel, Nature Photon. 3, 706 (2009).

[9] M. Macovei, J. Evers, C. H. Keitel, Phys. Rev. A 72, 063809 (2005).

[10] A. Auffeves, D. Gerace, S. Portolan, A. Drezet, M. Santos, New Jr. of Phys. 13, 093020 (2011).

[11] D. Norris, A. Cimmarusti, L. Orozco, P. BarberisBlostein, H. Carmichael, Phys. Rev. A 86, 053816 (2012).

[12] M. Macovei, C. H. Keitel, Phys. Rev. B 75, 245325 (2007); L. Jin, J. Evers, M. Macovei, Phys. Rev. A 84,
043812 (2011)

[13] A. Allevi, S. Olivares, M. Bondani, Phys. Rev. A 85, 063835 (2012).

[14] E. del Valle, A. Gonzalez-Tudela, F. Laussy, C. Tejedor, M. Hartmann, Phys. Rev. Lett. 109, 183601 (2012).

[15] D. A. Antonosyan, T. V. Gevorgyan, G. Yu. Kryuchkyan, Phys. Rev. A 83, 043807 (2011).

[16] J. Pritchard, C. Adams, K. Molmer, Phys. Rev. Lett. 108, 043601 (2012).

[17] F. Bariani, Y. Dudin, T. Kennedy, A. Kuzmich, Phys. Rev. Lett. 108, 030501 (2012).

[18] B. R. Mollow, Phys. Rev. 188, 1969 (1969).

[19] G. S. Agarwal, Phys. Rev. A 41, 2886 (1990).

[20] M. Koch, C. Sames, M. Balbach, H. Chibani, A. Kubanek, K. Murr, T. Wilk, G. Rempe, Phys. Rev. Lett. 107, 023601 (2011).

[21] R. Tan, G.-x. Li, Z. Ficek, Phys. Rev. A 78, 023833 (2008).

[22] X. Guo, Sh. Lü, Zh. Ren, Jr. of Phys. B: At., Mol. Opt. Phys. 43, 225401 (2010).

[23] D. F. V. James, Fort. Phys. 48, 823 (2000). 